\title{
Fallopian Tube Perforation
}

National Cancer Institute

\section{Source}

National Cancer Institute. Fallopian Tube Perforation. NCI Thesaurus. Code C78294.

A rupture of the fallopian tube wall due to tubal pregnancy, or other traumatic or pathologic processes. 